\title{
Application of Network Big Data on User Experience Application Model
}

\author{
Chun-Hui Chen* \\ Education Technology Center \\ Guangdong University of Foreign Studies \\ Guangzhou, China \\ 247031690@qq.com
}

\author{
Ke-Jing $\mathrm{Li}$ \\ School of Economics \& Trade \\ Guangdong University of Foreign Studies \\ Guangzhou, China \\ ljh@oamail.gdufs.edu.cn
}

\begin{abstract}
With the strong representativeness, objectiveness, efficiency, and timeliness, online big data helps companies build the best user experience based on understanding users and continues to bring infinite value to the company. This study starts with the development needs of user experience application under the impact of big data, combines the features of user experience disciplines with the characteristics of big data, and identifies two possible paths for optimizing user experience by big data. Through designing a research model, the study deals with Big Data containing three small loops and a big loop of user experience. The study investigates the data resource challenge of user experience research under network Big Data.
\end{abstract}

Keywords-Psychology, Network Big Data, User experience, Research model.

\section{INTRODUCTION}

Psychology is the science that studies human psychological and behavioral activities to realize the prediction, intervention, and control of human psychological behavior. Its ultimate goal is to promote the progress of human society. The era of great revolution in science and technology poses new challenges to the "human-centered" research of psychology. Glamorous traditional psychology has gradually shown its drawbacks and limitations. In this context, various marginal disciplines that intersect with psychology emerge as the times require, and user experience is one of them. User experience initially originated from human-computer interaction design. It is a new applied science generated by the integration of psychology, computer science, physiology, design, engineering and other disciplines. It is subjective psychological experience of users in the process of using a product or service. Understanding and grasping user experience directly serve the product design and improvement [1].

Big Data, also known as massive data, refers to the huge amount of data that cannot be intercepted, managed, processed, and organized in a reasonable amount of time by human labor to become human interpretable information [2]. The development of computer and information science technologies such as data mining makes it possible to efficiently process and analyze mass human behavior data, thus laying the technical foundation for massive data mining [3]. For companies, using technology to analyze big data can reduce cost, increase efficiency, and improve user experience. For example, JD.com understands users through accurate data analysis and mining. Its accurate recommendation enables conversion rate of intelligent store up to $20 \%$, big data forecast enables surprise delivery time of 3 minutes and 52 seconds, big data judging users' needs enables JIMI's (JD.com's artificial intelligence customer service robots) answer satisfaction rate beyond the manual, creating an ultimate user experience. In addition, the use of big data to analyze user behavior can bring a wide range of users with a strong stickiness. For example, Netflix, a company that produces Houses of Cards, used network big data to collect and analyze the user's viewing, selection, and replay and stop behaviors (including user autonomous behaviors and network restrictions) in real time. To understand the user's preferences through the feedback of these behavior data, the company modeled to predict user expectations. These measures maintained the user's activity, greatly enhanced the video ratings. These examples demonstrate that enterprise's induction and analysis of user behavior data has promoted quantitative research and qualitative improvement of user experience.

\section{CURRENT NETWORK USER EXPERIENCE RESEARCH RESULTS AND LIMITATIONS}

\section{A. Data Volume and Source Defects, Credibility}

In the traditional research methods for user experience, the experimenters often spend a lot of manpower and material resources on the sample collection, but still facing the problem of insufficient sample or lack of representativeness. Guo Yu et al. (2018) constructed a user experience evaluation system of online community knowledge consumption to fully excavate the knowledge needs of online community users and improve the quality of network community knowledge products [4]. However, the study only selected 10 active users to conduct interviews. Although it has some reference significance for the ecological construction of users in the Internet community, due to the small data coverage, the reference value remains to be assessed. Big data technology faces global users, providing more accurate data can make up for the accuracy of analysis results.

\section{B. Traditional Research Methods are Scattered and Lack of Integrity and Multifaceted Nature}

Traditionally used research methods for user experience include focus groups, telephone interviews, questionnaire surveys, email feedback, field studies, usability studies, etc. Short-term measurement methods are insufficient to cover all aspects of user experience and cannot reflect the dynamic variability of user experience. However, the long-term 
measurement method can measure all aspects of the users well, but it takes a long time, hard to operate, obtains mostly qualitative data, and data analysis and processing are more troublesome [5]. Big data technology can solve the shortcomings of various traditional methods to a certain degree by virtue of the characteristics of direct data acquisition, realtime implementation tracking and personalized response of user experience through multiple data sources.

\section{Relevant Model Method is Narrow in Scope and Difficult to Apply across Industries}

The research of user experience is an area that every company that has its own product actively concerns and different companies have self-contained methods. Various highly-specific indicators are difficult for other industries to learn from. Yan Min et al. (2017) comprehensively considered four key quality indicators of web browsing, instant messaging, application download, and video services and 16 key performance indicators based on signaling monitoring data and proposed an evaluation method of mobile communication network user experience quality [6]. The user experience rating scale developed by Guo Fu et al. (2017) for mobile games is divided into six dimensions: feedback, immersion, challenge, and social mobility and control [7]. Jiang Libing et al. (2017) have constructed a user experience model of "a core value, four content structures, nine constituent elements, and two experience levels" of digital learning resources and expected to optimize the construction of digital learning resources [8]. From the research on the user experience of Internet products in recent years, it is not difficult to see that although the researchers have high enthusiasm for this promising area and the fruits are endless, their specific industry goals are not conducive to user experience research in the face of crossindustry products with different features, which limits the breadth of the model.

From the above analysis, it's reasonable to conclude that the characteristics of $4 \mathrm{~V}$ possessed by big data, namely, volume (great volume), variety (various modality), velocity (rapid generation), and value (great value but low density), can solve the defects of sampling and research methods to a certain degree. On the basis of this, it is necessary to propose a widely applicable user experience research model based on big data.

\section{BIG DATA FOR USER EXPERIENCE}

\section{A. Improve the Efficiency of $R \& D$ and Update}

On the one hand, the user experience is extremely subjective and it is the perception and thoughts of a person to a system. On the other hand, the user experience is dynamic and will change according to the user's usage and time. Because of these characteristics, traditionally the user experience is not easy to capture and measure. The use of big data to implement real-time monitoring on user experience, to master the user's core needs, and then to carry out user demand forecast, simplifies the procedure of development and testing user experience measurement tools of product development process. In addition, with the extension of human-computer interaction in the time and space dimensions, commonality and continuous traceability of network big data allow designers to increase control over the direction of product updates, such as applying
A/B tests and gray scale tests to determine if the product needs iteration. Of course, the most ideal system can identify and locate changes in user requirements in time and respond automatically and continuously, from detailed microinnovations to version iterations, which eliminate the need for manual intervention in the process, greatly increase the efficiency of satisfaction fulfillment and decreases the cost of input needed.

\section{B. Strengthen Personalized Design}

Under the background of social and economic development, in addition to the functional requirements of the product, users have grown the implicit psychological needs such as emotionality. Therefore, user-centered design begins to prevail and has become the focus of product designers. From the conception of customization to user-centeredness, to Aarron Walter's publication of "Designing for Emotion", it is a change in the wind direction that has occurred in just a few decades. Although human-centered design as a young concept is full of attractiveness, it is still at the initial and laboratory stage, and it still faces many obstacles before put into practice due to cost and technical issues. The appearance of big data technology undoubtedly solves the difficulty that the humanized design has faced in the past. The data resources contained in big data itself can be used to enhance the user experience and provide data conditions that can fully characterize users in a comprehensive manner for the user's personalized management [9]. The synergy between personalized design and data proves that the user experience must be enhanced by relying on big data. Conversely, user experience is an important indicator of the ability of big data services, and user experience detection is a prerequisite for improving user experience [10].

\section{Research on The Construction OF UsER EXPERIENCE MODEL BASED ON INTERNET Big DATA}

\section{A. The Proposal of the Model}

The study of human psychology focuses on internal psychological activities and explicit behavior. Corresponding to the field of user experience, it can be divided into two sections, the attitudes towards specific product or system and the behaviors shown in actual operations. Under such classification logic, the research data of user experience comes from two aspects of attitude and behavior. Different from the research logic of hypothesis test of traditional psychology, big data is based on data analysis and the research logic is posterior. The results analyzed and summed up from the data is equivalent to the alternative hypothesis that needs to be confirmed, namely "data-driven", which greatly improves the efficiency and possibility of ultimately obtaining correct inferences [1].

The entire data analysis process of analyzing the resulting data and arriving at the decision is an attempt by the user experience researchers to make hypotheses. Based on the assumptions of data induction, the next step for user experience designers is to design and implement specific "experiments"improvements and updates of systems or products. Sometime after the commissioning, the experimenter will obtain the updated attitude and behavior data as experimental results, and then compare the results with previous hypotheses to verify the 
alternative hypothesis, thus correcting the decision making it more effective. The revised decision-making model will then be used to analyze new attitudes and behavior data to propose new hypotheses... so repeated, the system will enter a circulating, self-optimizing closed-loop with the support of big data, as illustrated in Fig.2. To further illustrate the operation principle of the model, the big cycle model is divided into three small loops: Attitude-Decision Ring, Behavior-Decision Ring, and Attitude-Behavior Ring.

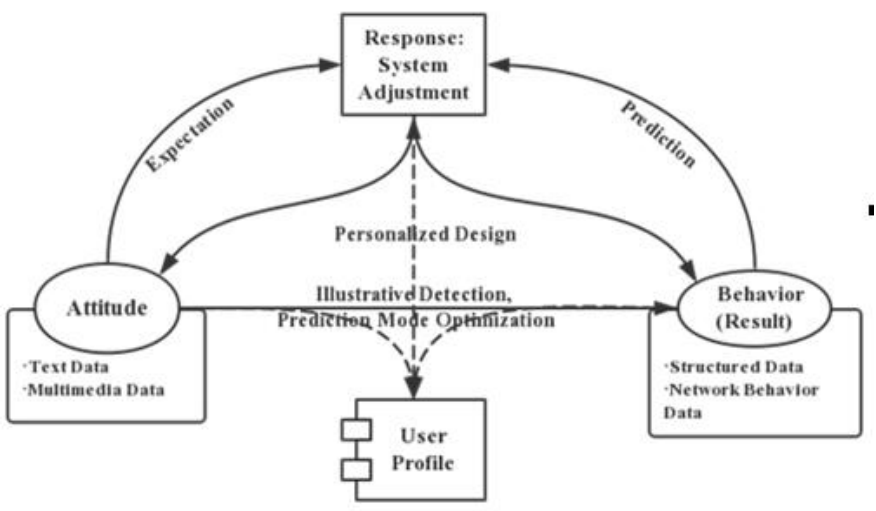

Fig. 1 User Experience Model based on Internet Big Data.

\section{B. Attitude-Decision Ring}

Attitude information mainly refers to the user's direct and indirect views and opinions on products or systems. Since attitudes are mostly created and published actively by users, they have unstructured features that are common in text and multimedia data. The user attitude can be divided into three categories. The first category is the users' tendentious evaluation on existing products or systems. It directly reflects the customer's preferences and is called the Recommendation category, which helps the researcher to adjust the current settings. The second category is new ideas derived spontaneously by the users from the current product or system. It is called Creative category. The viewpoints presented by the users from the perspective of the planner can become a researcher's inspiration and enlightenment for the destructive innovation of user experience. The third category includes the views users publish on the platform that are weakly related to specific products but strongly related to their own characteristics or social hot spots. They are called General category. If researchers can find and interpret this implicit information in a good way, there may be a great success in user experience design.

Among the above three types of user attitudes, unusual valuable but often overlooked in past research is creative attitudes, which directly expresses the users' expectations and can play a powerful guiding role in the adjustment of the system after the extraction of big data. The researcher responds to and adjusts the system according to the direction of the attitude, and after that tests the effectiveness of the adjustment by retesting attitudes, forming a closed loop. This cycle is conducive to the realization of a truly user-centric product design, as shown in Fig. 3.

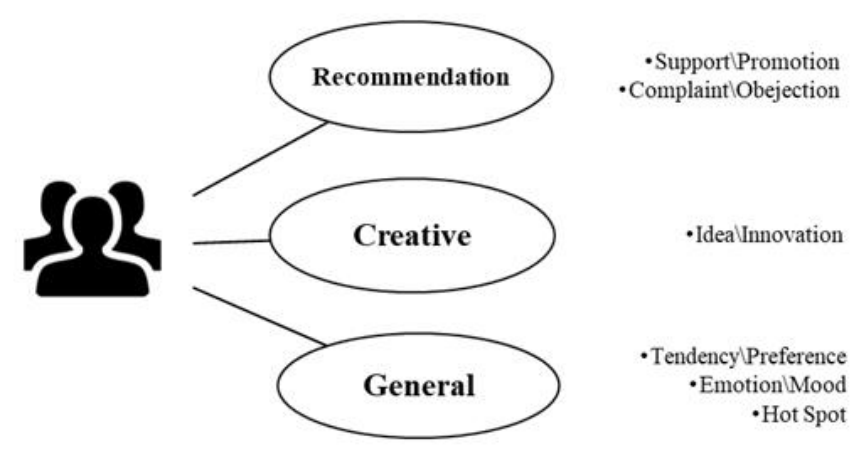

Fig. 2 User Attitude

\section{Behavior-Decision Ring}

The nature of user behavior, which is different from indirect attitude, has a direct impact on the company's interests. Therefore, user behavior data is also called result data. Behavior data is usually composed of two types of data, structured data, and users' network usage behavior data. Structured data is an indicator traditionally valued by enterprises, but as unstructured data, network behavior data especially server log files is easy to be ignored by companies. If we can make good use of this scattered but substantive behavioral information, use the big data approach to conduct an in-depth analysis of the behavior, and predict past behaviors that may occur in the past, we may be able to adjust the system to encourage the expected behaviors while suppressing the behaviors with negative effects. As with the Attitude-Decision Ring, constantly updated behavioral data and ongoing system adjustments can form a closed loop for automated operations.

Due to the strong directness and high correlation of behavioral influence, the goal of analyzing and studying user attitudes still falls on predicting and controlling user behaviors that have a direct impact on the benefits of the enterprise. For profitable companies, the main concern may be the user's purchase and activity. For non-profit companies, the main concern may be the user's participation. The importance of user behavior is self-evident and attitude can be regarded as a mediating variable used to predict the final behavior in this sense. If the conditions are not allowed, the interpretability should not be excessively pursued. In the case of limited corporate resources, focusing on the Behavior-Decision Ring and reducing or abandoning the construction of other loops is preferable.

\section{Attitude-Behavior Ring}

Using big data to capture user attitudes can help companies better understand the psychological mechanisms behind user behavior, and thus more accurately grasp and predict user behaviors. One possible method is to compare differences in user behaviors with different attitudes through big data and establish links between behaviors and attitudes to explain the differences in behaviors of users with different attitudes. It is the job of the researchers to constantly compare the real-time attitude and behavior data to detect the degree of attitudes to behaviors and to optimize the attitude-behavior prediction model. It is noteworthy that there is a certain degree of difference in the relationship of attitudes and behaviors on 
different individuals. In order to improve the accuracy of prediction, we can consider modeling from the individual microcosmic point of view.

When the user experience designer grasps the user's attitude and behavior data at the same time, the personalized design mentioned in the previous article can be realized. Personalized design is based on the enterprise's understanding of its users. It establishes a database to store users' attitudes and behaviors that have been generated in the past and conducts an in-depth analysis of the user's data to draw an accurate portrait of the user. Big data will greatly enhance the insight of the researchers and designers on user characteristics and develop differentiated user-centered designs that meet the subjective needs of users.

\section{Future Research Prospect}

The same as any other analysis, the data source is always the premise of all big data processing. The scale of big data is large and the data quality affects the efficiency and accuracy of the algorithm. For the enterprise, compared to obtaining all the data generated by a user in one system, getting the user's data on other similar or heterogeneous platforms can more effectively promote the understanding of the user's overall network behavior. Currently, there are problems such as a single data model and insufficient openness in the data resource sharing system, which has led to the mismatch of data resources and user experience design decisions of smallest and medium size organizations. Network-based data resource sharing is just as traditional enterprises share their customer resources. Good strategic cooperation will achieve a multiplier effect. Information resources are the life line of the development in the current Internet era and in the future, relevant research institutions can seek further cooperation in data resources and enhance the efficiency and effectiveness of analysis.

\section{SUMMARY}

Big Data helps the user experience research walk out of the laboratory. Because of the outstanding predictability, flexibility, and feedback of the model it builds, big data has extremely strong application value. This paper constructs the theoretical model oriented to the enterprise of big data application on user experience design and development. Unlike the user experience multi-factor model with different indicators proposed by the traditional psychology, this model especially aims at the network scene and disassembles the user experience into attitudes and behaviors, which greatly simplifies the analysis of the researchers, expands the breadth of the model's application, and satisfies the research needs of the user experience in the Internet world. In the age of big data, future user experience designers are more like data artists.

\section{REFERENCES}

[1] T. S. Zhu, J. Y. Wang, N. Zhao, X. Q. Liu, "Reform on Psychological Research in Big Data Age”, Journal of Xinjiang Normal University (Philosophy and Social Sciences), China, vol 04, pp. 100-107+2, 2015 (In Chinese)

[2] The Importance of (Big Data): A Definition. [online]. https://www.gartner.com/doc/2057415

[3] H. Chen, G. A. Le, M. Li, Y. H. Dong, "Computational social science: a common opportunity for Social Sciences and Information Science", Journal Of Southwest University (Social Sciences Edition), China, Vol.03, pp. 87-93, 2013. (In Chinese)

[4] Y. Guo, X. Q, Wang, M. Q. Yang, "Evaluation on Users'Experience of Knowledge Consumption in Network Community Based on Grounded Theory and BP Neural Network Analysis", Information Studies: Theory Application, China, vol. 03, pp. 117-122+141, 2018. (In Chinese)

[5] Y. Ding, F. Guo, M. C. Hu, F. L. Sun, “A Review of User Experience”, Industrial Engineering and Management, China, Vol. 04, pp. 92-97+114, 2014. (In Chinese)

[6] M. Yan, S. X. Gao, W. G. Yang, "Fuzzy Analytic Hierarchy Process Method for Evaluating Quality of Experience in Mobile Network", Journal of Network New Media, China, Vol. 03, pp. 1-7, 2017. (In Chinese)

[7] F. Guo, J. Y. Jiang, W. Lv, Y. Wang, “Development of a Scale for User Experience in Mobile Games and Construct Validation", Chinese Journal of Ergonomics, China Vol. 04, pp. 24-32, 2017. (In Chinese)

[8] L. B. Jiang, L. Y. Wan, Y. Yu, "Research on the User Experience Model in Utilizing Digital Learning Resources", Modern Education Technology Vol. 27, China, Number 3, pp. 85-92, 2017. (In Chinese)

[9] B. Kang, S. Q. Liu, "Management of the User Experience Based on the Big Data Analysis", Telecommunication Science, China, Vol.03, pp. 32-35, 2013. (In Chinese)

[10] J. Han, "Research on Some Key Technologies Of Big Data-as-a-service", doctor, Beijing University of Posts and Telecommunications, China, 2013. (In Chinese) 